\title{
SYNTHESIS, SPECTRAL ANALYSIS AND ANTIBACTERIAL EVALUATION OF 5-SUBSTITUTED-1,3,4- OXADIAZOL-2-YL 4-(4-METHYLPIPERIDIN-1-YLSULFONYL)BENZYL SULFIDES
}

\author{
AZIZ-UR-REHMAN ${ }^{a}$ *, SAMREEN AHTZAZ ${ }^{a}$, MUHAMMAD ATHAR ABBASI ${ }^{a}$, SABAHAT ZAHRA SIDDIQUI ${ }^{a}$, \\ SHAHID RASOOL ${ }^{a}$ IRSHAD AHMAD
}

\author{
${ }^{a}$ Department of Chemistry, Government College University, Lahore-54000, Pakistan. \\ ${ }^{b}$ Department of Pharmacy, The Islamia University of Bahawalpur, Bahawalpur-63100, Pakistan.
}

\begin{abstract}
Owing to valuable biological activities of 1,3,4-oxadiazole, sulfamoyl and piperidine functionalities, some new 1-(4-\{[(5-substituted-1,3,4-oxadiazol-2-yl) thio]methyl $\}$ benzene sulfonyl)-4-methylpiperidine (6a-o) derivatives have been introduced. The target molecules were synthesized from different aralkyl/aryl carboxylic acids, 1a-o, through a series of steps. First the compounds, 1a-o, were converted to heterocyclic 1,3,4-oxadiazole nucleophiles, 4a-0. Second an electrophile as 1-(4-bromomethylbenzenesulfonyl)-4-methylpiperidine (5) was synthesized from 4-methylpiperidine. Finally the target compounds, 6a-o, were prepared by reacting 4a-o with $\mathbf{5}$ in DMF and LiH. The final compounds were structurally elucidated by spectral data of IR, ${ }^{1} \mathrm{H}-\mathrm{NMR}$ and EI-MS. All the compounds were screened for their antibacterial evaluation and found to exhibit valuable results.
\end{abstract}

Keywords: 1,3,4-oxadiazole, 4-methylpiperidine, antibacterial activity, sulfonamide, ${ }^{1} \mathrm{H}-\mathrm{NMR}$, EI-MS.

\section{INTRODUCTION}

New diseases have been discovered and also the commercial drugs have become ineffective against the old ones. So a large work has to be performed in this regard in search of new more potent drug molecules. Organic synthesis along with other fields of research is continuously being used for drug development program and hence designing new drug candidates ${ }^{1,2}$.

Oxadiazole is a class of heterocyclic organic compounds that is known because of its magnificent therapeutic potential ${ }^{3}$. 1,3,4-Oxadiazole moieties display an inclusive variety of biological and pharmacological activities, for example, anti-cancer, anti-oxidant, anti-tubercular ${ }^{4-6}$. Besides anti-bacterial and anti-fungal properties, they have other biological properties such as anticonvulsant, anti-inflammatory and anti-anxiety ${ }^{7,8}$. Sulfonamides, antimicrobial agents, are synthetically formed from sulfanilic acid ${ }^{9-11}$. They are highly putative antibiotic due to their cost effectiveness and stable chemical activity ${ }^{12}$. Correspondingly, they exhibit antibacterial, antiviral, diuretic, anti-carbonic anhydrase and anti-thyroid activities ${ }^{13,14}$. The piperidine and its derivatives have also acquired substantial prominence to medicinal chemistry because of the wide variety of their biological and pharmaceutical properties ${ }^{15}$. Apart from this, piperidine nucleus is playing fundamental role as agrochemicals, anti-depressants, analgesics, anti-bacterial, anti-fungicidal, anti-histaminic, anti-HIV and anti-cancer agents ${ }^{16,17}$.

The valuable biological results of nominated functionalities prompted us to synthesize some new molecules bearing all these functionalities together in a single molecule and further to evaluate their pharmacological behavior regarding antibacterial activity. In continuation of previous attempts by our group in this regard ${ }^{18,19}$, the presented compounds with some new chemical entities were synthesized and subjected to antibacterial screening against certain strains of Gram-negative and Gram-positive bacteria.

\section{EXPERIMENTAL}

\subsection{General}

All the chemicals (Merck \& Alfa Aesar) and analytical grade solvents were purchased through local suppliers. Melting points were taken on Griffin and George melting point apparatus by open capillary tube method. TLC plate $\mathrm{F}_{256} 20 \times 20 \mathrm{~cm}$ coated with silica gel was used to detect the purity of the synthesized compounds, developed by different ratios of $n$-Hexane and EtOAc as solvent system. UV lamp was used to visualize the TLC. With the help of $\mathrm{KBr}$ pellet method, IR spectrum was recorded by using Jasco-320-A spectrophotometer. ${ }^{1} \mathrm{H}-\mathrm{NMR}$ spectra were recorded on Bruker spectrometers at frequency $400 \mathrm{MHz}$, taken in deuterated chloroform, indicating chemical shifts value in ppm and taking TMS as reference standard.

2.2. Procedure for the synthesis of different ethyl esters (2a-0)

The different organic acid $(0.02 \mathrm{~mol} ; \mathbf{1 a - 0})$ was taken in $200 \mathrm{~mL}$ round bottom flask. $20 \mathrm{~mL}$ absolute ethanol $(99 \%)$ was added to the flask and stirred for ten minutes to homogenize the reaction contents. Later on, $2.5 \mathrm{~mL}$ concentrated sulphuric acid, an activator, was added and reaction contents were refluxed for 3-4 hours. Thin layer chromatography was the technique used to monitor the completion of reaction by using 1:4 ratio of $n$-hexane and ethyl acetate as mobile phase. After maximum completion, reaction mixture was treated with $10 \%$ aqueous $\mathrm{Na}_{2} \mathrm{CO}_{3}(\mathrm{pH} 9-10)$ to neutralize the reaction mixture and to remove unreacted free acid. To get the pure esters, solvent extraction of neutralized reaction mixture was performed by $50 \mathrm{~mL}$ of $\mathrm{CHCl}_{3}$ in a separating funnel for 3-4 times. After that $\mathrm{CHCl}_{3}$ layer was separated from the lower density aqueous layer and was distilled by using rotary evaporator to acquire ethyl esters.

2.3. Procedure for the synthesis of different aralkyl/aryl acid hydrazides (3a-o)

The ethyl esters $(0.15 \mathrm{~mol} ; \mathbf{2} \mathbf{a}-\mathbf{0})$ were taken in $250 \mathrm{~mL}$ round bottom flask. About $30 \mathrm{~mL}$ of methanol was added to the flask and homogenized by stirring at room temperature. Hydrazine hydrate $(80 \% ; 0.15 \mathrm{~mol})$ was introduced to the flask drop wise and the reaction apparatus was refluxed for 5-6 hrs. Reaction progress was confirmed by thin layer chromatography. $n$-Hexane and ethyl acetate $(3: 2)$. At the end of reaction, excess of solvent was evaporated and residue was poured into the ice cold water. Precipitates of acid hydrazides were filtered, washed with water and dried. Re-crystallization of acid hydrazides was performed by using methanol.

2.4. Procedure for the synthesis of 5-substituted-1,3,4-oxadiazol-2thiols (4a-0)

The aralkyl/aryl acid hydrazides $(0.01 \mathrm{~mol}$; 3a-o) were dissolved in $20 \mathrm{~mL}$ absolute $\mathrm{C}_{2} \mathrm{H}_{5} \mathrm{OH}$ in a $250 \mathrm{~mL}$ of round bottom flask. Solid $\mathrm{KOH}(0.02 \mathrm{~mol})$ and carbon disulphide $(0.02 \mathrm{~mol})$ were added to reaction flask. The reaction contents were refluxed for 4-5 hrs with continuous stirring. $\mathrm{H}_{2} \mathrm{~S}$ was evolved during the completion of reaction. Completion of reaction was confirmed by thin layer chromatography by using $n$-hexane and ethyl acetate (3:2) as mobile phase to develop a chromatogram. After confirmation, cold distilled water was added to the flask contents along with the addition of dilute $\mathrm{HCl}(\mathrm{pH} \mathrm{4-5).}$ Precipitates of title compounds were filtered, washed with distilled water and dried. To obtain the pure products, precipitates were recrystallized from methanol.

2.5. Synthesis of 1-(4-bromomethylbenzenesulfonyl)-4methylpiperidine (5)

4-Methylpiperidine $(0.0126 \mathrm{~mol}$; a) was taken in an iodine flask. Aqueous sodium carbonate solution $(15 \%)$, a weak base, was added to maintain a $\mathrm{pH}$ of 9. Small amount methanol $(2-3 \mathrm{~mL})$ and 4-bromomethylbenzenesulfonyl chloride $(0.0126 \mathrm{~mol} ; \mathbf{b})$ were added to the mixture pinch by pinch. The mixture was shaken vigorously and set to stir for 1 hour till precipitation. White precipitates of 1-(4-bromomethylbenzenesulfonyl)-4-methylpiperidine were filtered, washed with distilled water and dried. The synthesized electrophile was re-crystallized from methanol and used for further reaction.

2.6. General procedure for the $S$-substituted derivatives (6a-0)

The molecules 4a-o $(0.0006 \mathrm{~mol})$ were homogenized in $5-10 \mathrm{~mL}$ of $N, N$-dimethylformamide in a round bottom flask and then $(0.0006 \mathrm{~mol})$ of lithium hydride was added along with stirring. After half hour stirring, 1-(4-bromomethylbenzenesulfonyl)-4-methylpiperidine (0.0006 mol) was 
poured into homogeneous solution and further stirred for 4-6 hours. After confirmation of reaction completion by Thin Layer Chromatography, ice cold distilled water was introduced into reaction contents along with 2-3 drops of aq. $\mathrm{NaOH}$. The mixture was gently shaken and precipitates of title compounds were acquired through filtration. Precipitates were washed with distilled water and dried. All the compounds were re-crystallized from methanol.

2.7. Physical and spectral data of synthesized compounds 2.7.1. 1-(4-\{[(5-Phenyl-1,3,4-oxadiazol-2-yl)thio|methyl\} benzenesulfonyl)-4-methylpiperidine (6a)

White amorphous solid; M.P: $109-111{ }^{\circ} \mathrm{C}$; Yield: $85 \%$; M.F: $\mathrm{C}_{21} \mathrm{H}_{23} \mathrm{~N}_{3} \mathrm{O}_{3} \mathrm{~S}_{2}$; M.W: 429.12; IR ( $\left.\mathrm{KBr}, v, \mathrm{~cm}^{-1}\right): 3030(\mathrm{C}-\mathrm{H}$ str. of ar. ring), $1634(\mathrm{C}=\mathrm{N}$ str. of oxadiazole ring), $1524\left(\mathrm{max}=\mathrm{C}\right.$ ar. str.), $1330\left(-\mathrm{SO}_{2}\right.$ str.), 1226, 1059 (C-O-C bond str.), 701 (C-Cl bond str.), 616 (C-S bond str.); ${ }^{1} \mathrm{H}-\mathrm{NMR}\left(\mathrm{CDCl}_{3}, 400\right.$ MHz): $\delta$ (ppm) 7.98 (d, $\left.J=7.2 \mathrm{~Hz}, 2 \mathrm{H}, \mathrm{H}-2^{\prime} \& \mathrm{H}-6^{\prime}\right), 7.70$ (d, $J=8.0 \mathrm{~Hz}, 2 \mathrm{H}$, H-2" \& H-6"), 7.62 (d, $J=8.0 \mathrm{~Hz}, 2 \mathrm{H}, \mathrm{H}-3 "$ " \& H-5"), 7.48 (m, 3H, H-3' to H-5'), 4.50 (s, 2H, H-7"), 3.70 (d, $\left.J=10.8 \mathrm{~Hz}, 2 \mathrm{H}, \mathrm{H}_{e q}-2^{\prime \prime \prime} \& \mathrm{H}_{e q}-6^{\prime \prime \prime}\right), 2.25$ (t, $\left.J=11.2 \mathrm{~Hz}, 2 \mathrm{H}, \mathrm{H}-2^{\prime \prime \prime} \& \mathrm{H}-6^{\prime \prime \prime}\right), 1.55\left(\mathrm{~d}, J=9.2 \mathrm{~Hz}, 2 \mathrm{H}, \mathrm{H}-3^{e q}\right.$ \& $\left.\& \mathrm{H}-5^{\prime \prime \prime}\right)$, $1.35\left(\mathrm{~d}, J=12.4 \mathrm{~Hz}, 2 \mathrm{H}, \mathrm{H}-3^{\prime \prime \prime} \& \mathrm{H}-5^{\prime \prime \prime}\right), 1.19-1.00$ (m, $\left.1 \mathrm{H}, \mathrm{H}-4{ }^{\prime \prime \prime}\right), 0.90$ (d, $J$ $\left.=4.0 \mathrm{~Hz}, 3 \mathrm{H}, \mathrm{CH}_{3}-7^{\prime \prime \prime}\right)$; EI-MS: $m / z$ 429 (4\%)[M] $]^{+}, 267$ (62\%), $251(89 \%), 177$ (18\%), 119 (4\%), $103(100 \%), 98$ (88\%), 77 (69\%).

2.7.2. 1-[4-(\{[5-(2-Chlorophenyl)-1,3,4-oxadiazol-2-yl]thio $\}$ methyl) benzenesulfonyl]-4-methylpiperidine ( $6 \mathrm{~b})$

Light pink amorphous solid; M.P: $90-92{ }^{\circ} \mathrm{C}$; Yield: $76 \%$; M.F: $\mathrm{C}_{2} \mathrm{H}_{22} \mathrm{ClN}_{3} \mathrm{O}_{3} \mathrm{~S} ; \mathrm{M} . \mathrm{W}: 463.08$; IR $\left(\mathrm{KBr}, v, \mathrm{~cm}^{-1}\right): 3031$ (C-H str. of ar. ring), 1635 ( $\mathrm{C}=\mathrm{N}$ str. of oxadiazole ring), $1523\left(\mathrm{C}=\mathrm{C}\right.$ ar. str.), $1329\left(-\mathrm{SO}_{2}\right.$ str. $), 1227$, 1059 (C-O-C bond str.), 704 (C-Cl bond str.), 615 (C-S bond str.); ${ }^{1} \mathrm{H}-\mathrm{NMR}$ $\left(\mathrm{CDCl}_{3}, 400 \mathrm{MHz}\right): \delta(\mathrm{ppm}) 7.90\left(\mathrm{~d}, J=7.2 \mathrm{~Hz}, 1 \mathrm{H}, \mathrm{H}-6^{\prime}\right), 7.72(\mathrm{~d}, J=8.0 \mathrm{~Hz}$, $2 \mathrm{H}, \mathrm{H}-2 "$ \& H-6"), 7.61 (d, $J=7.6 \mathrm{~Hz}, 2 \mathrm{H}, \mathrm{H}-3 "$ \& H-5"), 7.53 (d, $J=7.6 \mathrm{~Hz}$, $\left.1 \mathrm{H}, \mathrm{H}-3^{\prime}\right), 7.45\left(\mathrm{t}, J=7.6 \mathrm{~Hz}, 1 \mathrm{H}, \mathrm{H}-4^{\prime}\right), 7.38\left(\mathrm{t}, J=7.6 \mathrm{~Hz}, 1 \mathrm{H}, \mathrm{H}-5^{\prime}\right), 4.50$ (s, $\left.2 \mathrm{H}, \mathrm{H}-7^{\prime \prime}\right), 3.75$ (d, $\left.J=9.6 \mathrm{~Hz}, 2 \mathrm{H}, \mathrm{H}_{e q}-2^{\prime \prime \prime} \& \mathrm{H}_{e}-6^{\prime \prime \prime}\right), 2.25$ (d, $J=10.8 \mathrm{~Hz}, 2 \mathrm{H}$, $\mathrm{H}-2$ "' \& H -6"'), 1.55 (d, $\left.J=8.8 \mathrm{~Hz}, 2 \mathrm{H}, \mathrm{H}-3^{\prime \prime \prime} \& \mathrm{H}-5{ }^{\prime \prime \prime}\right), 1.35$ (d, $J=12.4$ $\left.\mathrm{Hz}, 2 \mathrm{H}, \mathrm{H}_{a x}{ }^{a x} \mathrm{-}^{\prime \prime} \& \mathrm{H}_{a x}-5^{\prime \prime \prime}\right), 1.17-1.05\left(\mathrm{~m}, 1 \mathrm{H}, \mathrm{H}-4{ }^{\prime \prime \prime}\right), 0.90$ (d, $J=4.0 \mathrm{~Hz}, 3 \mathrm{H}$, $\left.\mathrm{CH}_{3}-7^{\prime \prime \prime}\right)$; EI-MS $m / z: 463(4 \%)\left[\mathrm{M}^{+}\right], 302(67.3 \%), 251(77.9 \%), 212(17.8 \%)$, $153(26.8 \%), 137(4.2 \%), 116(100 \%), 98(88.8 \%)$.

2.7.3. 1-[4-(\{[5-(3-Chlorophenyl)-1,3,4-oxadiazol-2-yl]thio\}methyl) benzenesulfonyl]-4-methylpiperidine (6c)

White amorphous solid; M.P: $107-109 \quad{ }^{\circ} \mathrm{C}$; Yield: $72 \%$; M.F: $\mathrm{C}_{21} \mathrm{H}_{22} \mathrm{ClN}_{3} \mathrm{O}_{3} \mathrm{~S}_{2} ; \mathrm{M} . \mathrm{W}: 463.08$; IR ( $\left.\mathrm{KBr}, v_{\max } \mathrm{cm}^{-1}\right): 3031$ (C-H str. of ar. ring), $1635\left(\mathrm{C}=\mathrm{N}\right.$ str. of oxadiazole ring), $1523\left(\mathrm{ma}=\mathrm{C}\right.$ ar. str.), $1327\left(-\mathrm{SO}_{2}\right.$ str. $), 1227$, 1059 (C-O-C bond str.), 708 (C-Cl bond str.), 615 (C-S bond str.); ${ }^{1} \mathrm{H}-\mathrm{NMR}$ $\left(\mathrm{CDCl}_{3}, 400 \mathrm{MHz}\right): \delta$ (ppm) 7.95 (s, $\left.1 \mathrm{H}, \mathrm{H}-2^{\prime}\right), 7.85$ (d, $\left.J=7.6 \mathrm{~Hz}, 1 \mathrm{H}, \mathrm{H}-6^{\prime}\right)$, 7.72 (d, $\left.J=7.6 \mathrm{~Hz}, 2 \mathrm{H}, \mathrm{H}-2^{\prime \prime} \& \mathrm{H}-6^{\prime \prime}\right), 7.61$ (d $J=7.6 \mathrm{~Hz}, 2 \mathrm{H}, \mathrm{H}-3 "$ " \& H-5"), 7.48 (d, $\left.J=7.6 \mathrm{~Hz}, 1 \mathrm{H}, \mathrm{H}-4^{\prime}\right), 7.42$ (t, $\left.J=7.6 \mathrm{~Hz}, 1 \mathrm{H}, \mathrm{H}-5^{\prime}\right), 4.51$ (s, 2H, H-7' '), $3.76\left(\mathrm{~d}, J=10.4 \mathrm{~Hz}, 2 \mathrm{H}, \mathrm{H}-2^{\prime \prime \prime} \& \mathrm{H}-6^{\prime \prime \prime}\right), 2.23\left(\mathrm{t}, J=10.4, \mathrm{~Hz}, 2 \mathrm{H}, \mathrm{H}-2^{\prime \prime}\right.$ ' \& $\left.\mathrm{H}_{a x}-6^{\prime \prime \prime}\right), 1.67\left(\mathrm{~d}, J=7.6 \mathrm{~Hz}, 2 \mathrm{H}, \mathrm{H}_{e q}{ }^{-2}{ }^{\prime \prime \prime} \& \mathrm{H}_{e q}-5{ }^{\prime \prime \prime}\right), 1.31(\mathrm{~d}, J=12.4 \mathrm{~Hz}, 2 \mathrm{H}$, $\left.\mathrm{H}_{a x}-3^{a x} \& \mathrm{H}_{a x}-5^{\prime \prime \prime}\right), 1.16-1.00$ (m, $\left.1 \mathrm{H}, \mathrm{H}-4{ }^{\prime \prime \prime}\right), 0.93$ (d, $J=4.0 \mathrm{~Hz}, 3 \mathrm{H}, \mathrm{CH}_{3}-7^{\prime}$ "); EI-MS $m / z$ : $463(3 \%)\left[\mathrm{M}^{+}\right], 302(60.3 \%), 251(100 \%), 212(16.8 \%), 153$ (16.8\%), 137 (3.2\%), $116(88 \%), 98(87.3 \%)$.

2.7.4.

1-(4-\{[(5-Benzyl-1,3,4-oxadiazol-2-yl)thio]methyl\} benzenesulfonyl)-4-methylpiperidine (6d)

Light brown amorphous solid; M.P: $63-65{ }^{\circ} \mathrm{C}$; Yield: $83 \%$; M.F: $\mathrm{C}_{22} \mathrm{H}_{25} \mathrm{~N}_{3} \mathrm{O}_{3} \mathrm{~S}_{2}$; M.W: 443.13; IR ( $\mathrm{KBr}, v_{\max }, \mathrm{cm}^{-1}$ ): 3043 (C-H str. of ar. ring), 1637 ( $\mathrm{C}=\mathrm{N}$ str. of oxadiazole ring $), 1529$ ( $\mathrm{C}=\mathrm{C}$ ar. str.), 1349 (-SO str.), 1228, 1051 (C-O-C bond str.), 619 (C-S bond str.); ${ }^{1} \mathrm{H}-\mathrm{NMR}$ ( $\left.\mathrm{CDCl}_{3}, 400 \mathrm{MHz}\right): \delta$ (ppm) 7.65 (d, $J=8.0 \mathrm{~Hz}, 2 \mathrm{H}, \mathrm{H}-2 " \& \mathrm{H}-6 "), 7.50$ (d $J=8.0 \mathrm{~Hz}, 2 \mathrm{H}, \mathrm{H}-3 "$ " \& H-5"), 7.35-7.19 (m, 5H, H-2' to H-6'), 4.53 (s, 2H, H-7"), 4.21 (s, 2H, H-7'), $3.71\left(\mathrm{~d}, J=10.8 \mathrm{~Hz}, 2 \mathrm{H}, \mathrm{H}_{e q}-2^{2 \prime \prime} \& \mathrm{H}_{e q}-6^{\prime \prime \prime}\right), 2.18\left(\mathrm{t}, J=11.2 \mathrm{~Hz}, 2 \mathrm{H}, \mathrm{H}_{q}-2^{\prime \prime \prime}\right.$ \& $\left.\mathrm{H}-6^{\prime \prime \prime}\right), 1.59\left(\mathrm{~d}, J=7.6 \mathrm{~Hz}, 2 \mathrm{H}, \mathrm{H}-3^{e q} \& \mathrm{H}-5^{\prime \prime \prime}\right), 1.33(\mathrm{~d}, J=12.4 \mathrm{~Hz}, 2 \mathrm{H}, \mathrm{H}-$ 3"' \& $\mathrm{H}_{a x}-5$ "') $^{\circ}, 1.17-1.02\left(\mathrm{~m}, 1 \mathrm{H}, \mathrm{H}^{e q}-4 " '\right), 0.95$ (d, $\left.J=4.0 \mathrm{~Hz}, 3 \mathrm{H}, \mathrm{CH}_{3}-7{ }^{\prime \prime \prime}\right)$; EIMS $m / z$ : $443(8 \%)\left[\mathrm{M}^{+}\right], 281(69.3 \%), 252(88.9 \%), 192(38.1 \%), 191$ (36.8\%), $162(23.3 \%), 133(3.4 \%), 117(100 \%), 91(60.6 \%), 98(85.3 \%)$.

2.7.5. 1-[4-(\{[5-(4-Methylphenyl)-1,3,4-0xadiazol-2-yl]thio $\}$ methyl) benzenesulfonyl]-4-methylpiperidine (6e)

White amorphous solid; M.P: $83-85^{\circ} \mathrm{C}$; Yield: $80 \%$; M.F: $\mathrm{C}_{22} \mathrm{H}_{25} \mathrm{~N}_{3} \mathrm{O}_{3} \mathrm{~S}_{2}$; M.W: 443.58; IR ( $\left.\mathrm{KBr}, v, \mathrm{~cm}^{-1}\right): 3043(\mathrm{C}-\mathrm{H}$ str. of ar. ring), $1640(\mathrm{C}=\mathrm{N}$ str. of oxadiazole ring), 1537 ( $\mathrm{C}=\mathrm{C}$ ar. str.), 1359 ( $-\mathrm{SO}_{2}$ str.), 1232, 1064 (C-O-C bond str.), 623 (C-S bond str.); ${ }^{1} \mathrm{H}-\mathrm{NMR}\left(\mathrm{CDCl}_{3}, 400 \mathrm{MHz}\right): \delta$ (ppm) 7.84 (d, $\left.J=8.4 \mathrm{~Hz}, 2 \mathrm{H}, \mathrm{H}-2^{\prime} \& \mathrm{H}-6^{\prime}\right), 7.70$ (d, $\left.J=8.4 \mathrm{~Hz}, 2 \mathrm{H}, \mathrm{H}-3^{\prime} \& \mathrm{H}-5^{\prime}\right), 7.61$ (d, $\left.J=8.0 \mathrm{~Hz}, 2 \mathrm{H}, \mathrm{H}-2^{\prime \prime} \& \mathrm{H}-6^{\prime \prime}\right), 7.28$ (d, $J=7.6 \mathrm{~Hz}, 2 \mathrm{H}, \mathrm{H}-3 "$ \& H-5"), 4.51 (s, $2 \mathrm{H}, \mathrm{H}-7 "), 3.77$ (t, $\left.J=10.8 \mathrm{~Hz}, 2 \mathrm{H}, \mathrm{H}_{e q}-2^{2 \prime \prime} \& \mathrm{H}_{e q}-6^{\prime \prime \prime}\right), 2.27-2.22\left(\mathrm{~m}, 2 \mathrm{H}, \mathrm{H}_{a r}-2^{\prime}\right.$ "\& $\left.\mathrm{H}-6^{\prime \prime \prime}\right), 1.62\left(\mathrm{~d}, J=7.6 \mathrm{~Hz}, 2 \mathrm{H}, \mathrm{H}^{e q}-3{ }^{\prime \prime} \& \mathrm{H}^{e q}-5{ }^{\prime \prime \prime}\right), 1.38(\mathrm{~d}, J=12.4 \mathrm{~Hz}, 2 \mathrm{H}$, $\mathrm{H}_{-1} 3^{\prime \prime \prime} \&$ H $\left.-5^{\prime \prime \prime}\right), 1.21-1.11\left(\mathrm{~m}, 1 \mathrm{H}, \mathrm{H}-4{ }^{\prime \prime \prime}\right), 0.97$ (d, $J=4.0 \mathrm{~Hz}, 3 \mathrm{H}, \mathrm{CH}_{3}-7^{\prime}$ "); EI-MS $m / z$ : $443(4 \%)\left[\mathrm{M}^{+}\right], 281(60.3 \%), 251(100 \%), 191(16.8 \%), 133$
(3.2\%), 117 (98.6\%), 91 (67.6\%), 98 (87.3\%).

2.7.6. 1-[4-(\{[5-(2-Methoxyphenyl)-1,3,4-oxadiazol-2-yl]thio\}methyl) benzene sulfonyl]-4-methylpiperidine (6f)

Brown crystalline solid; M.P: $104-106^{\circ} \mathrm{C}$; Yield: $80 \%$; M.F: $\mathrm{C}_{22} \mathrm{H}_{25} \mathrm{~N}_{3} \mathrm{O}_{4} \mathrm{~S}_{2}$; M.W: 459.13; IR ( $\left.\mathrm{KBr}, v_{\max }, \mathrm{cm}^{-1}\right): 3052(\mathrm{C}-\mathrm{H}$ str. of ar. ring), $1642(\mathrm{C}=\mathrm{N}$ str. of oxadiazole ring), $1534(\mathrm{C}=\mathrm{C}$ ar. str.), 1332 (-SO, str.), 1226, 1055 (C-O-C bond str.), 618 (C-S bond str.); ${ }^{1} \mathrm{H}-\mathrm{NMR}\left(\mathrm{CDCl}_{3}, 400 \mathrm{MHz}\right): \delta$ (ppm) 7.85 (d, $J$ $\left.=7.2 \mathrm{~Hz}, 1 \mathrm{H}, \mathrm{H}-6^{\prime}\right), 7.71\left(\mathrm{~d}, J=8.4 \mathrm{~Hz}, 2 \mathrm{H}, \mathrm{H}-2^{\prime \prime} \& \mathrm{H}-6^{\prime \prime}\right), 7.63(\mathrm{~d}, J=8.0 \mathrm{~Hz}$, 2H, H-3" \& H-5"), 7.47 (t, $\left.J=7.2 \mathrm{~Hz}, 1 \mathrm{H}, \mathrm{H}-5^{\prime}\right), 6.50$ (t, $\left.J=8.0 \mathrm{~Hz}, 1 \mathrm{H}, \mathrm{H}-4^{\prime}\right)$, $6.60\left(\mathrm{~d}, J=8.0 \mathrm{~Hz}, 1 \mathrm{H}, \mathrm{H}-3^{\prime}\right), 4.56\left(\mathrm{~s}, 2 \mathrm{H}, \mathrm{H}-7^{\prime \prime}\right), 3.92\left(\mathrm{~s}, 3 \mathrm{H},-\mathrm{OCH}_{3}\right), 3.71(\mathrm{~d}$, $\left.J=11.2 \mathrm{~Hz}, 2 \mathrm{H}, \mathrm{H}-2^{\prime \prime \prime} \& \mathrm{H}-6^{\prime \prime \prime}\right), 2.23\left(\mathrm{t}, J=11.2 \mathrm{~Hz}, 2 \mathrm{H}, \mathrm{H}-2^{\prime \prime \prime} \& \mathrm{H}-6^{\prime \prime}\right.$ '), $1.69\left(\mathrm{~d}, J=7.6 \mathrm{~Hz}, 2 \mathrm{H}, \mathrm{H}_{e q}^{e q} 3^{\prime \prime \prime} \& \mathrm{H}_{e q}-5^{\prime \prime \prime}\right), 1.37\left(\mathrm{~d}, J=12.4 \mathrm{~Hz}, 2 \mathrm{H}, \mathrm{H}_{a x}^{a x}-3^{\prime \prime}\right.$ \& H -5"'), 1.12-0.99 (m, $\left.1 \mathrm{H}, \mathrm{H}-4{ }^{e \prime \prime}\right), 0.91$ (d, $\left.J=4.0 \mathrm{~Hz}, 3 \mathrm{H}, \mathrm{CH}_{3}-7^{\prime \prime \prime}\right)$; EI-MS $m / z: 459(5 \%)\left[\mathrm{M}^{+}\right], 297(60.3 \%), 251$ (87.9\%), 207 (16.8\%), 149 (3.2\%), 113 $(100 \%), 107(67.6 \%), 98(87.3 \%)$.

2.7.7. 1-[4-(\{[5-(2-Naphthylmethyl)-1,3,4-oxadiazol-2-yl]thio\}methyl) benzene sulfonyl]-4-methylpiperidine (6g)

White amorphous solid; M.P: $93-95^{\circ} \mathrm{C}$; Yield: $78 \%$; M.F: $\mathrm{C}_{26} \mathrm{H}_{27} \mathrm{~N}_{3} \mathrm{O}_{3} \mathrm{~S}_{2}$; M.W: 493.64; IR ( $\left.\mathrm{KBr}, v, \mathrm{~cm}^{-1}\right): 3036(\mathrm{C}-\mathrm{H}$ str. of ar. ring), $1638(\mathrm{C}=\mathrm{N}$ str. of oxadiazole ring), 1529 ( $\mathrm{C}=\mathrm{C}$ ar. str.), 1336 ( $-\mathrm{SO}_{2}$ str.), 1228, 1056 (C-O-C bond str.), 619 (C-S bond str.); ${ }^{1} \mathrm{H}-\mathrm{NMR}\left(\mathrm{CDCl}_{3}, 400 \mathrm{MHz}\right): \delta$ (ppm) 8.08 (d, $J$ $\left.=8.0 \mathrm{~Hz}, 1 \mathrm{H}, \mathrm{H}-4^{\prime}\right), 7.87\left(\mathrm{dd}, J=8.4,1.2 \mathrm{~Hz}, 1 \mathrm{H}, \mathrm{H}-8^{\prime}\right), 7.83$ (dd, $J=8.0,2.0$ $\left.\mathrm{Hz}, 1 \mathrm{H}, \mathrm{H}-5^{\prime}\right), 7.57$ (d, $\left.J=8.0 \mathrm{~Hz}, 2 \mathrm{H}, \mathrm{H}-2^{\prime \prime} \& \mathrm{H}-6^{\prime \prime}\right), 7.54$ (dd, $J=7.6,1.6 \mathrm{~Hz}$, $\left.1 \mathrm{H}, \mathrm{H}-3^{\prime}\right), 7.52\left(\mathrm{dt}, J=8.8,1.6 \mathrm{~Hz}, 1 \mathrm{H}, \mathrm{H}-7^{\prime}\right), 7.46-7.41\left(\mathrm{~m}, 1 \mathrm{H}, \mathrm{H}-6^{\prime}\right), 7.42(\mathrm{~s}$, $\left.1 \mathrm{H}, \mathrm{H}-1^{\prime}\right), 7.39$ (d, $\left.J=8.0 \mathrm{~Hz}, 2 \mathrm{H}, \mathrm{H}-3^{\prime \prime} \& \mathrm{H}-5^{\prime \prime}\right), 4.50$ (s, 2H, H-7"), 3.70 (d, $\left.J=11.2 \mathrm{~Hz}, 2 \mathrm{H}, \mathrm{H}_{e q}-2^{\prime \prime \prime} \& \mathrm{H}_{e q}-6^{\prime \prime \prime}\right), 2.25\left(\mathrm{t}, J=11.2 \mathrm{~Hz}, 2 \mathrm{H}, \mathrm{H}_{a x}-2^{\prime \prime \prime} \& \mathrm{H}_{a x}-6^{\prime \prime}\right.$ '), $1.57\left(\mathrm{~d}, J=8.8 \mathrm{~Hz}, 2 \mathrm{H}, \mathrm{H}_{e q}^{e q}-3^{\prime \prime \prime} \& \mathrm{H}_{e g}-5^{\prime \prime \prime}\right), 1.39$ (d, $J=12.4 \mathrm{~Hz}, 2 \mathrm{H}, \mathrm{H}_{o x}^{a x}-3^{\prime \prime \prime}$ \& H -5"'), 1.13-1.08 (m, $1 \mathrm{H}, \mathrm{H}-44^{\prime \prime \prime)}, 0.93$ (d, $J=4.0 \mathrm{~Hz}, 3 \mathrm{H}, \mathrm{CH}_{3}-7$ - $\left.^{\prime \prime}\right)$; EI-MS $m / z: 493(6 \%)[\mathrm{M}]^{+}, 332(70 \%), 251(78 \%), 241(27 \%), 183(4 \%), 167(100 \%)$, $141(78 \%), 98(77 \%)$.

2.7.8. 1-[4-(\{[5-(2,4-Dichlorophenyl)-1,3,4-oxadiazol-2-yl]thio\}methyl) benzene sulfonyl]-4-methylpiperidine $(6 \mathrm{~h})$

White amorphous solid; M.P: $109-111{ }^{\circ} \mathrm{C}$; Yield: $80 \%$; M.F: $\mathrm{C}_{21} \mathrm{H}_{21} \mathrm{Cl}_{2} \mathrm{~N}_{3} \mathrm{O}_{3} \mathrm{~S}$; M.W: 497.04; IR ( KBr, $\left.v_{\operatorname{mar}}, \mathrm{cm}^{-1}\right)$ : 3051 (C-H str. of ar. ring), $1653(\mathrm{C}=\mathrm{N}$ str. of oxadiazole ring $), 1532(\mathrm{C}=\mathrm{C}$ ar. str. $), 1367(-\mathrm{SO}$ str.), 1237, 1065 (C-O-C bond str.), 624 (C-S bond str.); ${ }^{1} \mathrm{H}-\mathrm{NMR}\left(\mathrm{CDCl}_{3}, 400\right.$ MHz): $\delta$ (ppm) 7.85 (d, $\left.J=8.8 \mathrm{~Hz}, 1 \mathrm{H}, \mathrm{H}-6^{\prime}\right), 7.72\left(\mathrm{~d}, J=8.0 \mathrm{~Hz}, 2 \mathrm{H}, \mathrm{H}-2^{\prime \prime} \&\right.$ H-6"), 7.61 (d $J=8.0 \mathrm{~Hz}, 2 \mathrm{H}, \mathrm{H}-3 "$ \& H-5"), 7.42 (s, 1H, H-3'), 7.37 (d, $J=$ $\left.8.0 \mathrm{~Hz}, 1 \mathrm{H}, \mathrm{H}-5^{\prime}\right), 4.53$ (s, 2H, H-7"), 3.72 (d, $J=10.8 \mathrm{~Hz}, 2 \mathrm{H}, \mathrm{H}_{e q}-2^{\prime \prime \prime} \& \mathrm{H}_{e q}-6$ "), $2.27\left(\mathrm{t}, J=11.2 \mathrm{~Hz}, 2 \mathrm{H}, \mathrm{H}-2^{\prime \prime \prime} \& \mathrm{H}-6^{\prime \prime \prime}\right), 1.55\left(\mathrm{~d}, J=8.8 \mathrm{~Hz}, 2 \mathrm{H}, \mathrm{H}-3{ }^{e q}\right.$ $\& \mathrm{H}_{e q}$-5"') $^{\prime \prime} 1.32\left(\mathrm{~d}, J=12.4 \mathrm{~Hz}, 2 \mathrm{H}, \mathrm{H}_{a x}{ }^{a x}{ }^{\prime \prime \prime} \& \mathrm{H}_{a x}-5^{\prime \prime \prime}\right), 1.14-1.06(\mathrm{~m}, 1 \mathrm{H}, \mathrm{H}-4$ "), 0.94 (d, $\left.J=4.0 \mathrm{~Hz}, 3 \mathrm{H}, \mathrm{CH}_{3}-7{ }^{\prime \prime \prime}\right)$; EI-MS $m / z: 497$ (6\%)[M+], 334 (70.3\%), 251 (77.9\%), 244 (26.8\%), 186 (26.8\%), 171 (4.2\%), 145 (100\%), 98 (88.3\%).

2.7.9. 1-[4-(\{[5-(2-Nitrophenyl)-1,3,4-oxadiazol-2-yl]thio\}methyl) benzenesulfonyl]-4-methylpiperidine (6i)

Dark brown amorphous solid; M.P: $59-61{ }^{\circ} \mathrm{C}$; Yield: 80\%; M.F: $\mathrm{C}_{21} \mathrm{H}_{22} \mathrm{~N}_{4} \mathrm{O}_{5} \mathrm{~S}$; M.W: 474.10; IR ( $\left.\mathrm{KBr}, v_{\max }, \mathrm{cm}^{-1}\right): 3037$ (C-H str. of ar. ring), $1639^{\circ}\left(\mathrm{C}=\mathrm{N}\right.$ str. of oxadiazole ring), $1522(\mathrm{~m}=\mathrm{C}$ ar. str. $), 1342$ ( $-\mathrm{SO}_{2}$ str.), 1232 , 1051 (C-O-C bond str.), 621 (C-S bond str.); ${ }^{1} \mathrm{H}-\mathrm{NMR}\left(\mathrm{CDCl}_{3}, 400 \mathrm{MHz}\right): \delta$ (ppm) 8.02 (d, $\left.J=7.2 \mathrm{~Hz}, 1 \mathrm{H}, \mathrm{H}-3^{\prime}\right), 7.90$ (d, $\left.J=7.2 \mathrm{~Hz}, 1 \mathrm{H}, \mathrm{H}^{3} 6^{\prime}\right), 7.79-7.73$ $\left(\mathrm{m}, 2 \mathrm{H}, \mathrm{H}-4^{\prime} \& \mathrm{H}-5^{\prime}\right), 7.72$ (d, $\left.J=8.0 \mathrm{~Hz}, 2 \mathrm{H}, \mathrm{H}-2^{\prime \prime} \& \mathrm{H}-6^{\prime \prime}\right), 7.60$ (d, $J=8.0 \mathrm{~Hz}$, 2H, H-3" \& H-5"), 4.52 (s, 1H, H-7"), 3.71 (d, $J=11.2 \mathrm{~Hz}, 2 \mathrm{H}, \mathrm{H}_{\text {eq }}-2^{\prime \prime \prime} \& \mathrm{H}_{\text {eq }}-6$ "), $2.25\left(\mathrm{t}, J=11.2 \mathrm{~Hz}, 2 \mathrm{H}, \mathrm{H}_{\text {or }} 2^{2 " \prime} \& \mathrm{H}_{o-}-6^{\prime \prime \prime}\right), 1.55\left(\mathrm{~d}, J=8.8 \mathrm{~Hz}, 2 \mathrm{H}, \mathrm{H}_{e q}{ }^{e q} 3^{\prime \prime \prime}\right.$ \& $\left.\mathrm{H}_{e q}-5^{\prime \prime \prime}\right), 1.35$ (d, $J=12.4 \mathrm{~Hz}, 2 \mathrm{H}, \mathrm{H}_{a x}{ }^{\prime \prime \prime}$ \& $\left.\mathrm{H}_{\alpha x}-5^{\prime \prime \prime}\right), 1.10-0.97$ (m, $1 \mathrm{H}, \mathrm{H}-4$ ' "), 0.90 (d, $\left.J=4.0 \mathrm{~Hz}, 3 \mathrm{H}, \mathrm{CH}_{3}-7^{\prime \prime \prime}\right)$; EI-MS $m / z: 474(5 \%)\left[\mathrm{M}^{+}\right], 312(43.0 \%)$, $251(87.4 \%), 222(1.9 \%), 164(2.3 \%), 148(2.7 \%), 98(100 \%), 122(9.6 \%)$.

2.7.10. 1-[4-(\{[5-(3-Nitrophenyl)-1,3,4-oxadiazol-2-yl]thio $\}$ methyl) benzenesulfonyl]-4-methylpiperidine $(6 \mathbf{j})$

Orange amorphous solid; M.P: $88-90^{\circ} \mathrm{C}$; Yield: 79\%; M.F: $\mathrm{C}_{21} \mathrm{H}_{22} \mathrm{~N}_{4} \mathrm{O}_{5} \mathrm{~S}_{2}$; M.W: 474.10; IR ( $\left.\mathrm{KBr}, v, \mathrm{~cm}^{-1}\right): 3030$ (C-H str. of ar. ring), $1637(\mathrm{C}=\mathrm{N}$ str. of oxadiazole ring), $1525^{\max }\left(\mathrm{C}=\mathrm{C}\right.$ ar. str.), 1327 ( $-\mathrm{SO}_{2}$ str.), 1223, 1059 (C-O-C bond str.), 616 (C-S bond str.); ${ }^{1} \mathrm{H}-\mathrm{NMR}\left(\mathrm{CDCl}_{3}, 400 \mathrm{MHz}\right): \delta(\mathrm{ppm}) 8.34$ (d, $\left.J=8.8 \mathrm{~Hz}, 1 \mathrm{H}, \mathrm{H}-4^{\prime}\right), 8.15$ (d, $\left.J=8.4 \mathrm{~Hz}, 1 \mathrm{H}, \mathrm{H}-6^{\prime}\right), 7.91-7.87$ (m, $\left.1 \mathrm{H}, \mathrm{H}-5^{\prime}\right)$, $7.73\left(\mathrm{~d}, J=7.2 \mathrm{~Hz}, 2 \mathrm{H}, \mathrm{H}-2^{\prime \prime} \& \mathrm{H}-6^{\prime \prime}\right), 7.70\left(\mathrm{~s}, 1 \mathrm{H}, \mathrm{H}-2^{2}\right), 7.62(\mathrm{~d}, J=7.6 \mathrm{~Hz}$, $2 \mathrm{H}, \mathrm{H}-3 "$ \& H-5"), 4.50 (s, 1H, H-7"), 3.71 (d, J=11.2 Hz, 2H, H $-2^{\prime \prime \prime} \& \mathrm{H}_{e q}-6^{\prime}$ "), $2.24\left(\mathrm{t}, J=11.2 \mathrm{~Hz}, 2 \mathrm{H}, \mathrm{H}_{o x}-2^{\prime \prime \prime} \& \mathrm{H}_{o x}-6^{\prime \prime \prime}\right), 1.54\left(\mathrm{~d}, J=8.8 \mathrm{~Hz}, 2 \mathrm{H}, \mathrm{H}_{e q}{ }^{e q}{ }^{\prime \prime}\right.$ \& H $\left.-5^{\prime \prime \prime}\right), 1.36$ (d, $\left.J=12.4 \mathrm{~Hz}, 2 \mathrm{H}, \mathrm{H}-3^{\prime \prime \prime} \& \mathrm{H}-5^{\prime \prime \prime}\right), 1.13-1.04(\mathrm{~m}, 1 \mathrm{H}, \mathrm{H}-4$ ' "), 0.97 (d, $\left.J=4.0 \mathrm{~Hz}, 3 \mathrm{H}, \mathrm{CH}_{3}-7^{\prime \prime \prime}\right)$; EI-MS $m / z: 474\left[\mathrm{M}^{+}\right](5 \%), 313(44.0 \%)$, $252(22.9 \%), 223(1.8 \%), 222(1.6 \%), 164(2.2 \%), 162(4.2 \%), 148(10.9 \%)$, $122(9.5 \%), 98(100 \%)$.

2.7.11. 1-[4-(\{[5-(4-Nitrophenyl)-1,3,4-oxadiazol-2-yl|thio\}methyl) benzenesulfonyl]-4-methylpiperidine (6k)

Yellow amorphous solid; M.P: $159-161^{\circ} \mathrm{C}$; Yield: $82 \%$; M.F: $\mathrm{C}_{21} \mathrm{H}_{22} \mathrm{~N}_{4} \mathrm{O}_{5} \mathrm{~S}_{2}$; 
M.W: 474.10; IR (KBr, $\left.v, \mathrm{~cm}^{-1}\right)$ : $3031(\mathrm{C}-\mathrm{H}$ str. of ar. ring), $1635(\mathrm{C}=\mathrm{N}$ str. of oxadiazole ring), $1523\left(\mathrm{C}=\mathrm{C}\right.$ ar. str.), 1327 ( $-\mathrm{SO}_{2}$ str.), 1227, 1059 (C-O-C bond str.), 615 (C-S bond str.); ${ }^{1} \mathrm{H}-\mathrm{NMR}\left(\mathrm{CDCl}_{3}, 400 \mathrm{MHz}\right): \delta$ (ppm) 8.35 (d, $\left.J=8.8 \mathrm{~Hz}, 2 \mathrm{H}, \mathrm{H}-3^{\prime} \& \mathrm{H}-5^{\prime}\right), 8.15$ (d, $\left.J=8.8 \mathrm{~Hz}, 2 \mathrm{H}, \mathrm{H}-2^{\prime} \& \mathrm{H}-6^{\prime}\right), 7.71$ (d, $J=8.0 \mathrm{~Hz}, 2 \mathrm{H}, \mathrm{H}-2 "$ \& H-6"), 7.63 (d, $J=8.0 \mathrm{~Hz}, 2 \mathrm{H}, \mathrm{H}-3 "$ \& H-5"), 4.62 (s, $1 \mathrm{H}, \mathrm{H}-7 "), 3.74$ (d, $J=10.8 \mathrm{~Hz}, 2 \mathrm{H}, \mathrm{H}$ - $\left.2^{\prime \prime \prime} \& \mathrm{H}-6^{\prime \prime \prime}\right), 2.25$ (t, $J=10.8 \mathrm{~Hz}, 2 \mathrm{H}$, $\left.\mathrm{H}_{a x}-2^{\prime \prime \prime} \& \mathrm{H}_{a x}-6^{\prime \prime \prime}\right), 1.65\left(\mathrm{~d}, J=9.2 \mathrm{~Hz}, 2 \mathrm{H}, \mathrm{H}_{e q}-3^{e q}+\mathrm{H}_{e q}-5^{\prime \prime \prime}\right), 1.30$ (d, $J=10.8$ $\left.\mathrm{Hz}, 2 \mathrm{H}, \mathrm{H}-3^{\prime \prime \prime} \& \mathrm{H}-5^{\prime \prime \prime}\right), 1.09-0.96\left(\mathrm{~m}, 1 \mathrm{H}, \mathrm{H}-4{ }^{\prime \prime \prime}\right), 0.91(\mathrm{~d}, J=4.0 \mathrm{~Hz}, 3 \mathrm{H}$, $\left.\mathrm{CH}_{3}-7^{\prime \prime \prime)}\right)$ EI-MS $m / z: 474(2 \%)\left[\mathrm{M}^{+}\right], 313(44.0 \%), 251$ (87.4\%), 223 (2.1\%), $164(2.5 \%), 148(2.5 \%), 98(100 \%), 122(9.9 \%)$.

2.7.12. 1-[4-(\{[5-(3-Aminophenyl)-1,3,4-oxadiazol-2-yl]thio\}methyl) benzenesulfonyl]-4-methylpiperidine (6I)

Half white amorphous solid; M.P: $100-102{ }^{\circ} \mathrm{C}$; Yield: $75 \%$; M.F $\mathrm{C}_{21} \mathrm{H}_{24} \mathrm{~N}_{4} \mathrm{O}_{3} \mathrm{~S}_{2}$; M.W: 444.13; IR ( $\left.\mathrm{KBr}, v_{\max }, \mathrm{cm}^{-1}\right): 3045$ (C-H str. of ar. ring), $1642(\mathrm{C}=\mathrm{N}$ str. of oxadiazole ring $), 1531(\mathrm{C}=\mathrm{C}$ ar. str. $), 1338\left(-\mathrm{SO}_{2}\right.$ str. $), 1233$, 1064 (C-O-C bond str.), 618 (C-S bond str.); ${ }^{1} \mathrm{H}-\mathrm{NMR}\left(\mathrm{CDCl}_{3}, 400 \mathrm{MHz}\right): \delta$ (ppm) 7.96 (d, $\left.J=7.2 \mathrm{~Hz}, 1 \mathrm{H}, \mathrm{H}-6^{\prime}\right), 7.69$ (d, $J=8.0 \mathrm{~Hz}, 2 \mathrm{H}, \mathrm{H}-2^{\prime \prime} \& \mathrm{H}-6^{\prime \prime}$ ), 7.62 (d, $J=8.0 \mathrm{~Hz}, 2 \mathrm{H}, \mathrm{H}-3 "$ " \& H-5"), 7.53 (t, $\left.J=9.1 \mathrm{~Hz}, 1 \mathrm{H}, \mathrm{H}-5^{\prime}\right), 7.53$ (s, $\left.1 \mathrm{H}, \mathrm{H}-2^{\prime}\right), 7.48$ (t, $\left.J=8.4 \mathrm{~Hz}, 1 \mathrm{H}, \mathrm{H}-4^{\prime}\right), 4.55$ (s, 1H, H-7"), $3.72(\mathrm{t}, J=10.8$ $\mathrm{Hz}, 2 \mathrm{H}, \mathrm{H}_{\rho q}-2 " '$ \& $\left.\mathrm{H}_{o-}-6 " '\right), 2.23-2.17$ (m, 2H, $\mathrm{H}_{a r}-2 " '$ \& $\left.\mathrm{H}_{a x}-6 " '\right), 1.68-1.63$ (m, $2 \mathrm{H}, \mathrm{H}$ of $\left.-3^{\prime \prime \prime} \& \mathrm{H}_{o q}-5^{\prime \prime \prime}\right), 1.35$ (d, $\left.J=12.4 \mathrm{~Hz}, 2 \mathrm{H}, \mathrm{H}_{o x}-3^{\prime \prime \prime} \& \mathrm{H}_{\alpha x}-5^{\prime \prime \prime}\right), 1.14-1.05$ $\left(\mathrm{m}, 1 \mathrm{H}, \mathrm{H}-4^{\prime \prime \prime}\right), 0.90$ (d, $\left.J=4.0 \mathrm{~Hz}, 3 \mathrm{H}, \mathrm{CH}_{3}-7^{\prime \prime \prime}\right)$; EI-MS $m / z: 445(6 \%)[\mathrm{M}]^{+}$, $282(59 \%), 251(87 \%), 192(16 \%), 134(5 \%), 118(100 \%), 98(89 \%), 92(70 \%)$.

2.7.13. 1-[4-(\{[5-(3,5-Dinitrophenyl)-1,3,4-oxadiazol-2-yl]thio\}methyl) benzene sulfonyl]-4-methylpiperidine (6m)

Light brown amorphous solid; M.P: $69-71{ }^{\circ} \mathrm{C}$; Yield: $83 \%$; M.F: $\mathrm{C}_{21} \mathrm{H}_{21} \mathrm{~N}_{5} \mathrm{O}_{7} \mathrm{~S}_{2}$; M.W: 519.09; IR ( $\left.\mathrm{KBr}, v_{\max } \mathrm{cm}^{-1}\right): 3042$ (C-H str. of ar. ring), $16366^{(\mathrm{C}=\mathrm{N}}$ str. of oxadiazole ring $), 1526(\mathrm{max}=\mathrm{C}$ ar. str. $), 1336\left(-\mathrm{SO}_{2}\right.$ str. $), 1229$, 1062 (C-O-C bond str.), 617 (C-S bond str.); ${ }^{1} \mathrm{H}-\mathrm{NMR}\left(\mathrm{CDCl}_{3}, 400 \mathrm{MHz}\right): \delta$ (ppm) 9.14 (s, 1H, H-4'), 9.10 (s, 2H, H-2' \& H-6'), 7.73 (d, J $=7.2 \mathrm{~Hz}, 2 \mathrm{H}$, H-2" \& H-6"), 7.60 (d, J=7.2 Hz, 2H, H-3" \& H-5"), 4.52 (s, 1H, H-7"), 3.71 (d, $\left.J=11.2 \mathrm{~Hz}, 2 \mathrm{H}, \mathrm{H}_{e q}-2^{\prime \prime \prime} \& \mathrm{H}_{e q}-6^{\prime \prime \prime}\right), 2.25$ (t, $J=11.2 \mathrm{~Hz}, 2 \mathrm{H}, \mathrm{H}_{a r}-2^{\prime \prime \prime} \& \mathrm{H}_{a x}-6^{\prime}$ "), 1.55 (d, $\left.J=8.8 \mathrm{~Hz}, 2 \mathrm{H}, \mathrm{H}-3^{\prime \prime \prime} \& \mathrm{H}-5^{\prime \prime \prime}\right), 1.34$ (d, $J=12.4 \mathrm{~Hz}, 2 \mathrm{H}, \mathrm{H}-3^{\prime \prime}$ ' \& $\left.\mathrm{H}_{a \gamma}-5^{\prime \prime \prime}\right), 1.11-1.03\left(\mathrm{~m}, 1 \mathrm{H}, \mathrm{H}-4{ }^{e \prime}\right), 0.94\left(\mathrm{~d}, J=4.0 \mathrm{~Hz}, 3 \mathrm{H}, \mathrm{CH}_{3}-7{ }^{\prime \prime \prime}\right)$; EIMS $m / z: 519(4 \%)\left[\mathrm{M}^{+}\right], 357(70.3 \%), 267(26.8 \%), 251(86.9 \%), 209(15.8 \%), 193$ (4.2\%), $167(100 \%), 98(67.3 \%)$.

2.7.14. 1-[4-(\{[5-(2-Chloro-3,5-dinitrophenyl)-1,3,4-oxadiazol-2-yl] thio\}methyl) benzenesulfonyl]-4-methylpiperidine (6n)

Dark brown amorphous solid; M.P: $98-100{ }^{\circ} \mathrm{C}$; Yield: $85 \%$; M.F: $\mathrm{C}_{21} \mathrm{H}_{20} \mathrm{ClN}_{5} \mathrm{O}_{7} \mathrm{~S}_{2} ; \mathrm{M} . \mathrm{W}: 553.54$; IR ( $\left.\mathrm{KBr}, v_{v}, \mathrm{~cm}^{-1}\right): 3030$ (C-H str. of ar. ring), $1634(\mathrm{C}=\mathrm{N}$ str. of oxadiazole ring $), 1524(\mathrm{C}=\mathrm{C}$ ar. str. $), 1330\left(-\mathrm{SO}_{2}\right.$ str. $), 1226$,
1059 (C-O-C bond str.), 616 (C-S bond str.); ${ }^{1} \mathrm{H}-\mathrm{NMR}\left(\mathrm{CDCl}_{3}, 400 \mathrm{MHz}\right)$ : $\delta$ (ppm) 9.05 (s, 1H, H-4'), 8.92 (s, 1H, H-6'), 7.80 (d, $J=7.6 \mathrm{~Hz}, 2 \mathrm{H}, \mathrm{H}-2^{\prime}$ ' \& H-6"), 7.65 (d, J=7.6 Hz, 2H, H-3" \& H-5"), 4.52 (s, 1H, H-7"), 3.7 (d, $\left.J=10.8 \mathrm{~Hz}, 2 \mathrm{H}, \mathrm{H}-2^{\prime \prime \prime} \& \mathrm{H}-6^{\prime \prime \prime}\right), 2.25\left(\mathrm{t}, J=11.2 \mathrm{~Hz}, 2 \mathrm{H}, \mathrm{H}-2^{\prime \prime \prime} \& \mathrm{H}-6^{\prime \prime}\right.$ '), $1.55\left(\mathrm{~d}, J=9.2 \mathrm{~Hz}, 2 \mathrm{H}, \mathrm{H}_{e q}^{e q} 3^{\prime \prime \prime} \& \mathrm{H}_{e q}-5^{\prime \prime \prime}\right), 1.35$ (d, $J=12.4 \mathrm{~Hz}, 2 \mathrm{H}, \mathrm{H}_{a x}^{a x}-3^{\prime \prime \prime}$

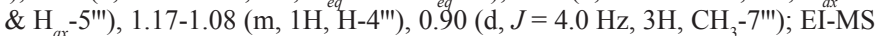
$m / z: 553(4 \%)[\mathrm{M}]^{+}, 267(62 \%), 251(89 \%), 177(18 \%), 119(4 \%), 103(100 \%)$, $98(88 \%), 77(69 \%)$.

2.7.15. 1-[4-(\{[5-(3-Pyridinyl)-1,3,4-oxadiazol-2-yl]thio\}methyl) benzenesulfonyl]-4-methylpiperidine (6o)

Dark brown amorphous solid; M.P: $102-104{ }^{\circ} \mathrm{C}$; Yield: $80 \%$; M.F: $\mathrm{C}_{20} \mathrm{H}_{22} \mathrm{~N}_{4} \mathrm{O}_{3} \mathrm{~S}_{2}$; M.W: 430.11; IR ( $\left.\mathrm{KBr}, v_{\text {max }}, \mathrm{cm}^{-1}\right)$ : 3036 (C-H str. of ar. ring), $1639(\mathrm{C}=\mathrm{N}$ str. of oxadiazole ring $), 1528(\mathrm{C}=\mathrm{C}$ ar. str. $), 1339\left(-\mathrm{SO}_{2}\right.$ str. $), 1234$, 1056 (C-O-C bond str.), 621 (C-S bond str.); ${ }^{1} \mathrm{H}-\mathrm{NMR}\left(\mathrm{CDCl}_{3}, 400 \mathrm{MHz}\right): \delta$ (ppm) 9.20 (s, 1H, H-2'), 8.77 (br.s, $\left.1 \mathrm{H}, \mathrm{H}-6^{\prime}\right), 8.36$ (d, $\left.J=7.6 \mathrm{~Hz}, 1 \mathrm{H}, \mathrm{H}-4^{\prime}\right)$, 7.72 (d, $\left.J=8.0 \mathrm{~Hz}, 2 \mathrm{H}, \mathrm{H}-2^{\prime \prime} \& \mathrm{H}-6 "\right), 7.60$ (d, $J=8.0 \mathrm{~Hz}, 2 \mathrm{H}, \mathrm{H}-3 "$ " \& H-5"), 7.54 (br.s, $\left.1 \mathrm{H}, \mathrm{H}-5^{\prime}\right), 4.53$ (s, $\left.1 \mathrm{H}, \mathrm{H}-7{ }^{\prime \prime}\right), 3.71$ (d, $J=11.2 \mathrm{~Hz}, 2 \mathrm{H}, \mathrm{H}-2^{\prime \prime \prime} \& \mathrm{H}-$ 6"'), $2.25\left(\mathrm{t}, J=11.2 \mathrm{~Hz}, 2 \mathrm{H}, \mathrm{H}_{\alpha x}-2^{\prime \prime \prime} \& \mathrm{H}_{o r}-6^{\prime \prime \prime}\right), 1.55\left(\mathrm{~d}, J=8.8 \mathrm{~Hz}, 2 \mathrm{H}, \mathrm{H}_{e-1}-3^{\prime \prime \prime}\right.$ \& H $\left.-5{ }^{\prime \prime \prime}\right), 1.35$ (d, $\left.J=12.4 \mathrm{~Hz}, 2 \mathrm{H}, \mathrm{H}-3^{\prime \prime \prime} \& \mathrm{H}_{a x}-5^{\prime \prime \prime}\right), 1.12-1.05$ (m, $1 \mathrm{H}, \stackrel{\text { H }}{ }-4^{\prime}$ "), $0.92\left(\mathrm{~d}, J=4.0 \mathrm{~Hz}, 3 \mathrm{H}, \mathrm{CH}_{3}-7 \mathrm{C}^{\prime \prime}\right)$; EI-MS $m / z: 430(3 \%)\left[\mathrm{M}^{+}\right], 268(60.3 \%)$, $251(87.9 \%), 178(16.8 \%), 120(3.2 \%), 104(100 \%), 98$ (87.3\%), 78 (67.6\%).

2.8. Antibacterial activity assay

The antibacterial activity was performed using sterile 96-wells microplates under aseptic environments. The method is based on the principle that microbial cell number increases as the microbial growth proceeds in a log phase of growth which results in increased absorbance of broth medium ${ }^{20,21}$.

\subsection{Statistical analysis}

All the calculations were accomplished after performing the experiment thrice and then analyzing statistically by Microsoft Excel 2010.

\section{RESULTS AND DISCUSSION}

Stimulated by significance of piperidine and 1,3,4-oxadiazole on the pharmaceutical and biological grounds, the synthesis of 1-(4-\{[(5-substituted1,3,4-oxadiazol-2-yl)thio]methyl benzenesulfonyl)-4-methylpiperidine (6a-o) was carried out by the protocol elaborated in scheme-1. The different aralkyl/ aryl groups are listed in Table-1. The detailed procedures are explicated in experimental section. The results for antibacterial activity are demonstrated as $\%$ inhibition and MIC (Minimum Inhibitory Concentration) values in Table-2 and Table-3, respectively.

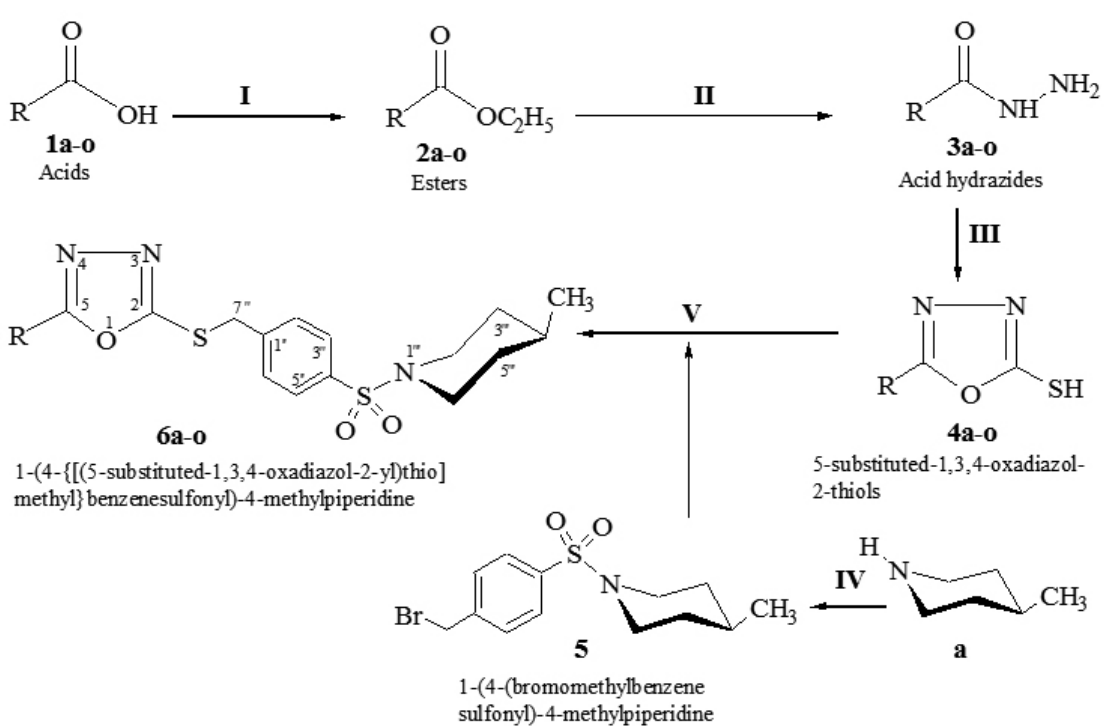

Scheme-1: Outline for the synthesis of 1-(4-\{[(5-substituted-1,3,4-oxadiazol-2-yl)thio] methyl $\}$ benzenesulfonyl)-4-methylpiperidine (6a-o). Reagents \& conditions: (I) EtOH, $\mathrm{H}_{2} \mathrm{SO}_{4}$, reflux for 3-4 hrs (II) $\mathrm{MeOH}, \mathrm{N}_{2} \mathrm{H}_{4}$, reflux for 5-6 hrs (III) EtOH, $\mathrm{KOH}, \mathrm{CS}_{2}$, reflux for 4-5 hrs (IV) 4-bromomethylbenzenesulfonyl chloride (b), $5 \% \mathrm{Na}_{2} \mathrm{CO}_{3}$ soln, $\mathrm{H}_{2} \mathrm{O}$, stir for $1 \mathrm{hr}$ (V) LiH, DMF, stir for 4-6 hrs. 
Table-1: Different aralkyl/aryl groups.

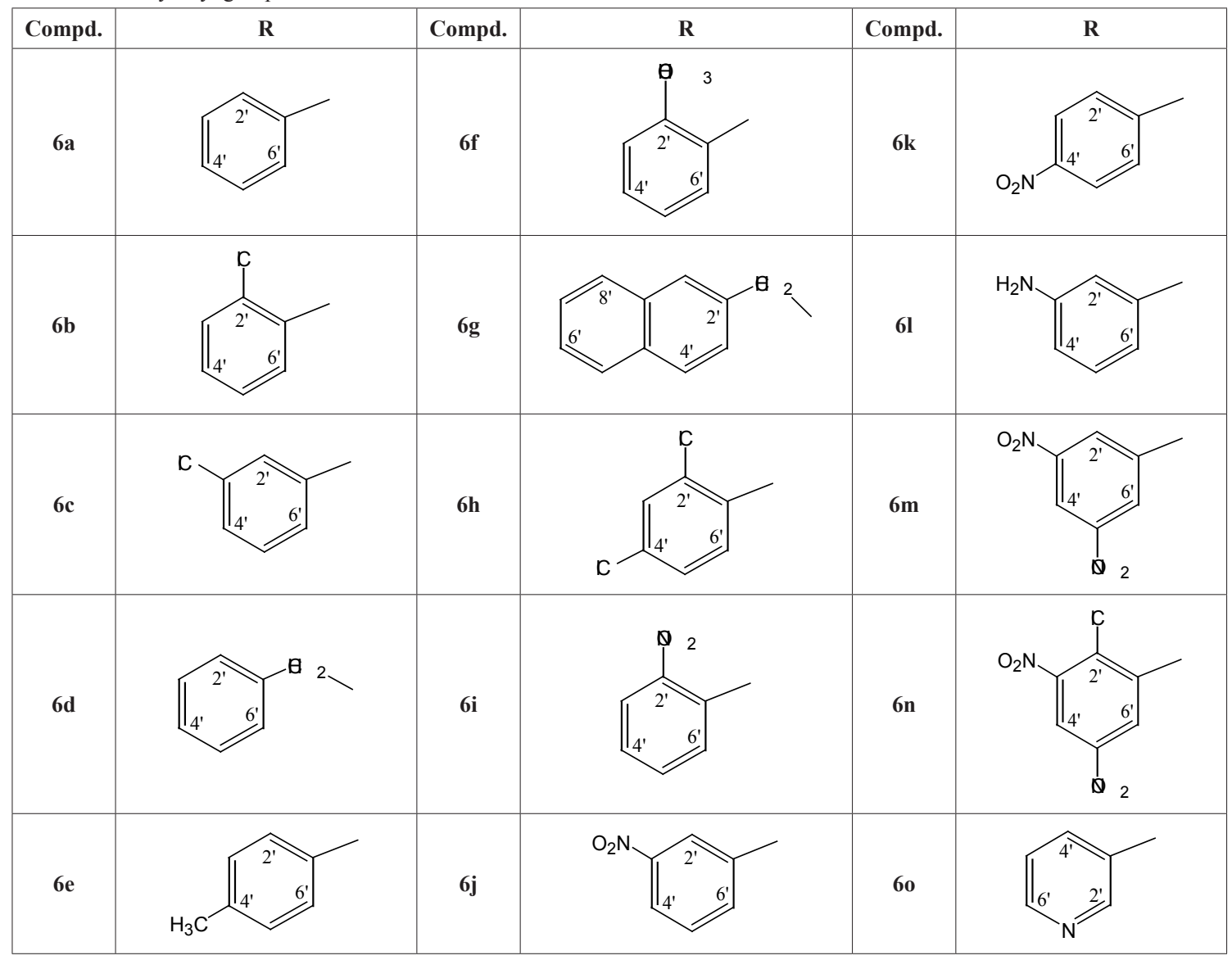

Table-2: \% Inhibition results for antibacterial activity of synthesized compounds.

\begin{tabular}{|c|c|c|c|c|c|}
\hline \multirow{2}{*}{ Compound } & \multicolumn{5}{|c|}{ \% INHIBITION } \\
\hline & S. typhi (-) & E. coli (-) & P. aeroginosa (-) & B. subtilis (+) & S. aureus (+) \\
\hline $6 a$ & $73.43 \pm 1.11$ & $75.34 \pm 0.20$ & $41.13 \pm 1.75$ & $78.75 \pm 0.80$ & $82.71 \pm 0.70$ \\
\hline $6 c$ & $68.71 \pm 0.22$ & $75.33 \pm 0.89$ & $60.50 \pm 0.11$ & $65.88 \pm 0.70$ & $71.86 \pm 0.50$ \\
\hline $6 d$ & $65.14 \pm 1.39$ & $73.10 \pm 0.47$ & $59.75 \pm 0.43$ & $72.13 \pm 0.75$ & $68.86 \pm 1.00$ \\
\hline $6 f$ & $59.57 \pm 1.09$ & $78.44 \pm 0.27$ & $62.13 \pm 0.55$ & $61.00 \pm 0.30$ & $65.43 \pm 0.60$ \\
\hline $6 \mathrm{~g}$ & $39.29 \pm 0.33$ & $65.11 \pm 0.60$ & $40.63 \pm 1.15$ & $38.38 \pm 0.55$ & $72.14 \pm 0.80$ \\
\hline $6 \mathrm{~h}$ & $65.14 \pm 0.61$ & $70.33 \pm 0.25$ & $49.50 \pm 0.35$ & $69.75 \pm 0.75$ & $71.14 \pm 0.95$ \\
\hline $6 \mathbf{i}$ & $65.29 \pm 0.39$ & $78.65 \pm 1.75$ & $64.88 \pm 1.05$ & $75.43 \pm 0.25$ & $53.25 \pm 0.35$ \\
\hline $6 \mathbf{j}$ & $69.29 \pm 0.72$ & $30.78 \pm 0.50$ & $57.38 \pm 0.15$ & $76.00 \pm 0.20$ & $65.50 \pm 0.10$ \\
\hline $6 n$ & $74.14 \pm 0.11$ & $67.22 \pm 0.70$ & $67.88 \pm 1.10$ & $73.00 \pm 0.40$ & $59.38 \pm 1.05$ \\
\hline 60 & $62.13 \pm 0.66$ & $68.56 \pm 1.22$ & $61.23 \pm 0.38$ & $74.25 \pm 0.79$ & $75.28 \pm 0.42$ \\
\hline Ciprofloxacin & $92.87 \pm 0.91$ & $92.27 \pm 0.64$ & $92.34 \pm 0.35$ & $91.63 \pm 0.05$ & $90.57 \pm 0.35$ \\
\hline
\end{tabular}


Table-3: MIC results for antibacterial activity of synthesized compounds

\begin{tabular}{|c|c|c|c|c|c|}
\hline \multirow{2}{*}{ Compound } & \multicolumn{5}{|c|}{ MIC ( $\boldsymbol{\mu m o l} / \mathbf{L})$} \\
\cline { 2 - 6 } & S. typhi (-) & $\boldsymbol{E}$ coli (-) & P. aeroginosa $(-)$ & B. subtilis (+) & S. aureus $(+)$ \\
\hline $\mathbf{6 a}$ & $10.32 \pm 0.64$ & $10.84 \pm 0.29$ & - & $10.55 \pm 0.94$ & $9.84 \pm 0.30$ \\
\hline $\mathbf{6 b}$ & $10.63 \pm 0.08$ & $10.33 \pm 0.36$ & $16.42 \pm 0.47$ & $12.64 \pm 0.60$ & $10.48 \pm 0.49$ \\
\hline $\mathbf{6 c}$ & $11.211 \pm 0.14$ & $10.21 \pm 0.65$ & $14.53 \pm 0.10$ & $12.57 \pm 0.29$ & $12.48 \pm 0.27$ \\
\hline $\mathbf{6 d}$ & $12.49 \pm 0.53$ & $11.16 \pm 0.40$ & $15.75 \pm 0.23$ & $11.84 \pm 0.18$ & $12.85 \pm 0.84$ \\
\hline $\mathbf{6 e}$ & $10.75 \pm 0.94$ & $10.18 \pm 0.49$ & $17.38 \pm 0.12$ & $15.28 \pm 0.09$ & $10.74 \pm 0.11$ \\
\hline $\mathbf{6 f}$ & $17.65 \pm 0.72$ & $9.43 \pm 0.15$ & $14.98 \pm 0.32$ & $15.73 \pm 0.22$ & $12.42 \pm 0.16$ \\
\hline $\mathbf{6 g}$ & - & $12.61 \pm 0.17$ & - & - & $10.87 \pm 0.10$ \\
\hline $\mathbf{6 h}$ & $10.27 \pm 0.68$ & $12.56 \pm 0.79$ & - & $10.37 \pm 0.17$ & $10.76 \pm 0.10$ \\
\hline $\mathbf{6 i}$ & $12.87 \pm 0.47$ & $10.38 \pm 0.81$ & $12.58 \pm 0.38$ & $10.29 \pm 0.09$ & $18.74 \pm 0.55$ \\
\hline $\mathbf{6 j}$ & $11.39 \pm 0.82$ & - & $14.29 \pm 0.05$ & $9.79 \pm 0.53$ & $12.74 \pm 0.48$ \\
\hline $\mathbf{6 k}$ & $12.47 \pm 0.71$ & $10.29 \pm 0.05$ & $16.31 \pm 0.94$ & $9.21 \pm 0.94$ & $15.38 \pm 0.33$ \\
\hline $\mathbf{6}$ & $19.21 \pm 0.68$ & $11.85 \pm 0.96$ & $14.29 \pm 0.30$ & $10.16 \pm 0.65$ & $12.76 \pm 0.90$ \\
\hline $\mathbf{6 m}$ & $11.72 \pm 0.31$ & $14.06 \pm 0.66$ & $14.76 \pm 0.29$ & $12.16 \pm 0.85$ & $13.12 \pm 0.81$ \\
\hline $\mathbf{6 n}$ & $10.52 \pm 0.18$ & $12.36 \pm 0.98$ & $12.40 \pm 0.54$ & $10.36 \pm 0.33$ & $16.98 \pm 0.64$ \\
\hline $\mathbf{6 0}$ & $11.23 \pm 0.67$ & $12.09 \pm 0.43$ & $14.62 \pm 0.91$ & $10.04 \pm 0.63$ & $10.77 \pm 0.51$ \\
\hline Ciprofloxacin & $\mathbf{7 . 8 3} \pm \mathbf{0 . 7 8}$ & $\mathbf{8 . 0 1} \pm \mathbf{0 . 1 2}$ & $\mathbf{7 . 9 8} \pm \mathbf{0 . 8 9}$ & $\mathbf{7 . 2 2} \pm \mathbf{0 . 6 7}$ & $\mathbf{7 . 0 0} \pm \mathbf{1 . 5 4}$ \\
\hline
\end{tabular}

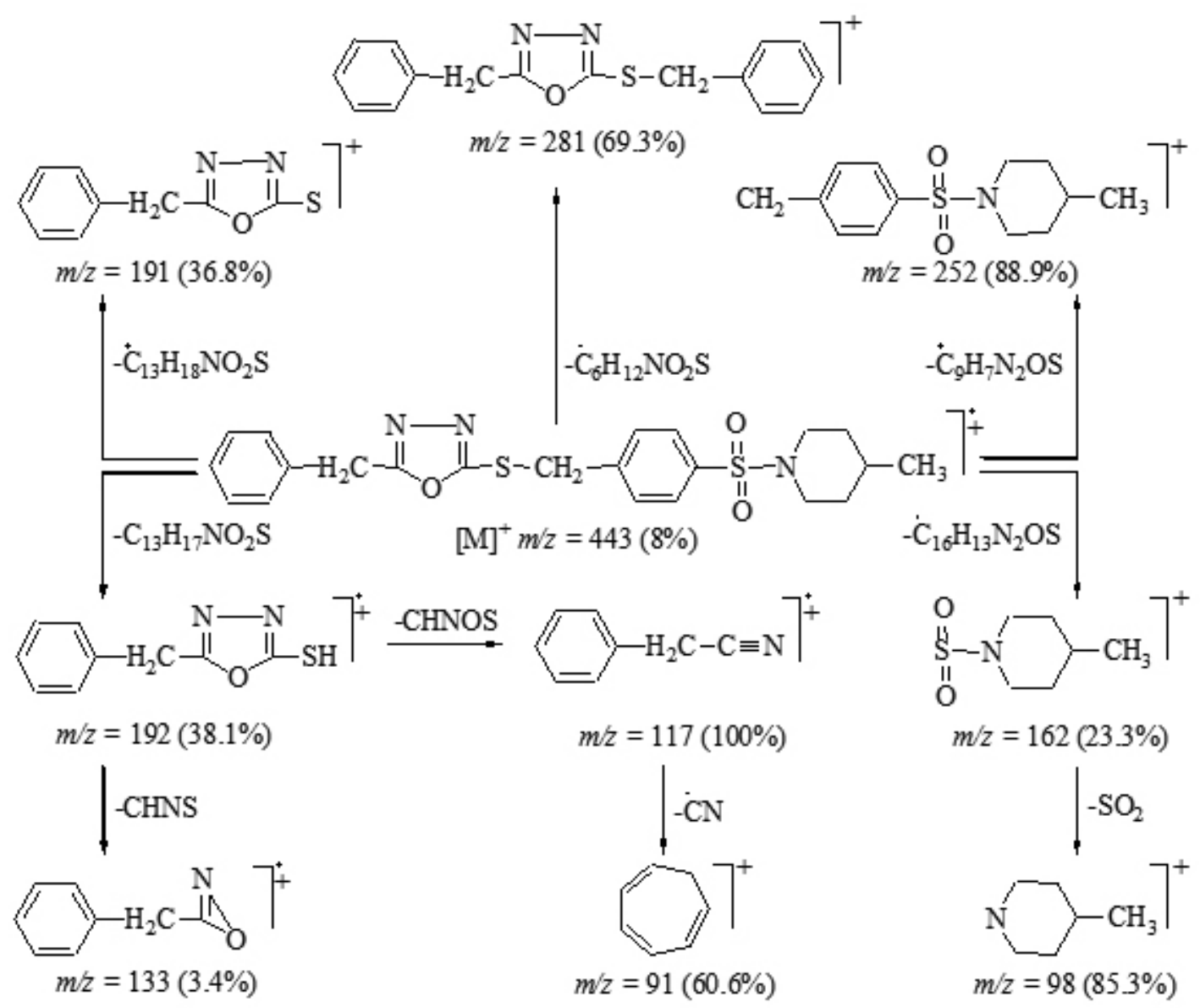

Figure-1: Proposed mass fragmentation pattern of 1-(4-\{[(5-benzyl-1,3,4-oxadiazol-2-yl)thio $]$ methyl $\}$ benzenesulfonyl)-4methylpiperidine (6d) 


\subsection{Chemistry}

The target molecules, 1-(4-\{[(5-substituted-1,3,4-oxadiazol-2-yl)thio] methyl $\}$ benzenesulfonyl)-4-methylpiperidine (6a-o), have been synthesized with reasonable yields from simple aralkyl/aryl carboxylic acids through a series of steps. First step is simple ethyl esterification, second one is about nucleophilic substitution of ethoxy of ester by hydrazine on stirring or refluxing, third one is the intermolecular cyclization of carbohydrazide into heterocyclic 1,3,4-oxadiazole. Solid $\mathrm{KOH}$ was used in this step to provide the basic media that facilitated the cyclization process. After addition of excess water, the $\mathrm{pH}$ was adjusted to low acidic one $(\mathrm{pH}=5-6)$ to acquire the product because of salt formation as potassium thioxide. Highly acidic $\mathrm{pH}$ has negative effect on yield because of further salt formation. The electrophile, 1-(4-bromomethylbenzenesulfonyl)-4-methylpiperidine (5) was synthesized by a facile method in a basic aqueous medium. The precipitates of electrophile were also acquired after adjusting $\mathrm{pH}$ to 5-6 and also too low $\mathrm{pH}$ was obviated. The last step comprised of the reaction of the synthesized electrophile and the nucleophilic 1,3,4-oxadiazol-2-thiols in a aprotic polar solvent along with an activator. All the compounds were structurally corroborated through spectral studies including IR, ${ }^{1} \mathrm{H}-\mathrm{NMR}$ and EIMS, given in experimental section.

Compound 6d was synthesized as light brown amorphous solid having yield $83 \%$ and melting point $63-65{ }^{\circ} \mathrm{C}$. Molecular formula was erected on the basis of EI-MS with molecular ion peak at $\mathrm{m} / \mathrm{z} 443$, forwarding to molecular formula as $\mathrm{C}_{22} \mathrm{H}_{25} \mathrm{~N}_{3} \mathrm{O}_{3} \mathrm{~S}_{2}$ which was also confirmed through protons in integration curves of its ${ }^{1} \mathrm{H}-\mathrm{NMR}$ spectrum. The IR spectrum supported the main functionalities by absorption bands at 3043 (for aromatic C-H), 1637 (for oxadiazole $\mathrm{C}=\mathrm{N}$ ), 1529 (for aromatic $\mathrm{C}=\mathrm{C}$ ), 1349 (for $\mathrm{S}=\mathrm{O}$ of sulfamoyl group), 1228, 1051 (for oxadiazole C-O-C) and 619 (for thiol C-S). In ${ }^{1} \mathrm{H}-\mathrm{NMR}$ spectrum, two signals were assigned to benzyl group: one multiplet at $\delta 7.35$ $7.19\left(\mathrm{~m}, 5 \mathrm{H}, \mathrm{H}-2^{\prime}\right.$ to $\left.\mathrm{H}-6^{\prime}\right)$ and one singlet at $\delta 4.21$ (s, 2H, H-7'). Nine signals were allocated to the 1-(4-Bromomethylbenzenesulfonyl)-4-methylpiperidine: two doublets in aromatic region at $\delta 7.65(\mathrm{~d}, J=8.0 \mathrm{~Hz}, 2 \mathrm{H}, \mathrm{H}-2 "$ \& H-6") and $7.50\left(\mathrm{~d} J=8.0 \mathrm{~Hz}, 2 \mathrm{H}, \mathrm{H}-3^{\prime \prime} \& \mathrm{H}-5 "\right)$; one singlet at $\delta 4.53$ (s, $\left.1 \mathrm{H}, \mathrm{H}-7 "\right)$; four doublets in aliphatic region at $\delta 3.71\left(\mathrm{~d}, J=10.8 \mathrm{~Hz}, 2 \mathrm{H}, \mathrm{H}-2^{\prime \prime \prime} \& \mathrm{H}-6^{\prime \prime \prime}\right)$, 1.59 (d, $\left.J=7.6 \mathrm{~Hz}, 2 \mathrm{H}, \mathrm{H}_{e q}-3^{3 \prime \prime} \& \mathrm{H}_{e q}-5^{\prime \prime \prime}\right), 1.33$ (d, J=12.4 Hz, $2 \mathrm{H}, \mathrm{H}_{a x}{ }^{e q}{ }^{-3} " '$ \& $\left.\mathrm{H}-5^{\prime \prime \prime}\right)$ and $0.95\left(\mathrm{~d}, J=4.0 \mathrm{~Hz}, 3 \mathrm{H}, \mathrm{CH}_{-}-7^{\prime \prime \prime}\right)$; one triplet at $\delta 2.18(\mathrm{t}, J=11.2$ $\mathrm{Hz}, 2 \mathrm{H}, \mathrm{H}_{a x}-2$ "' \& $\left.\mathrm{H}_{a x}-6^{\prime \prime \prime}\right)$; and one multiplet at $\delta 1.17-1.02$ (m, 1H, H-4"'). The afore mentioned data structurally confirmed the formation of new derivative as $\quad 1$-(4-\{[(5-benzyl-1,3,4-oxadiazol-2-yl)thio]methyl $\}$ benzenesulfonyl)-4methylpiperidine. The proposed mass fragmentation pattern of $\mathbf{6 d}$ is sketched out in Figure-1. Likewise the structures of other compounds were elucidated.

\subsection{Antibacterial Activity}

All the compounds were screened against $S$. typhi (-), E. coli (-), P. aeruginosa $(-), B$. subtilis $(+)$ and $S$. aureus $(+)$. The results are mentioned in Table 2 and Table 3 . The most of synthesized compounds demonstrated valuable results against almost all the strains. S. typhi (-), E. coli (-) and S. aureus $(+)$ were better inhibited by all the compounds except a few. The whole series remained the least active or inactive at all against $P$. aeruginosa (-). B. subtilis $(+)$ was moderately inhibited by the most ones and excellently by a few. The phenyl substituted compound, 6a, remained efficient inhibitor against all the strains except $P$. aeruginosa (-). The molecules bearing chlorophenyl groups, 6b \& 6c, possessed excellent potential against all the strains except moderate against $P$. aeruginosa (-). Compound, 1-[4-(\{[5-(2,4-Dichlorophenyl)1,3,4-oxadiazol-2-yl]thio methyl)benzenesulfonyl]-4-methylpiperidine $\quad(\mathbf{6 h})$ exhibited excellent inhibitory potential against $S$. typhi (-), B. subtilis (+) and $S$. aureus $(+)$ with MIC values of $10.27 \pm 0.68,10.37 \pm 0.17$ and $10.76 \pm 0.10 \mu \mathrm{mol} / \mathrm{L}$ relative to ciprofloxacin, a reference standard with MIC values of $7.83 \pm 0.78$, $7.22 \pm 0.67$ and $7.00 \pm 1.54 \mu \mathrm{mol} / \mathrm{L}$ respectively but against $E$. coli $(-)$, the inhibitory potential was not appreciable. It remained inactive at all against $P$. aeruginosa (-). The better activity of this molecule might be attributed to the presence of dichlorophenyl ring attached to bioactive oxadiazole ring. Compound, 1-[4-(\{[5-(2-Methoxyphenyl)-1,3,4-oxadiazol-2-yl]thio $\}$ methyl) benzenesulfonyl]-4-methylpiperidine (6f) demonstrated excellent inhibitory potential against $E$. coli (-) with MIC of $9.43 \pm 0.15 \mu \mathrm{mol} / \mathrm{L}$ relative to reference standard with $\mathrm{MIC}$ of $8.01 \pm 0.12 \mu \mathrm{mol} / \mathrm{L}$ but moderately low activities against the remaining bacterial strains. The compound $\mathbf{6 g}$ bearing naphthyl group demonstrated the least activity. The nitro substituted phenyl ring bearing compounds, $\mathbf{6} \mathbf{i}$ to $\mathbf{6 j}$, remained notably efficient against $E$. coli (-) and excellent inhibitor of $B$. subtilis $(+)$.

\section{CONCLUSION}

All the compounds were obtained in good yields and structurally corroborated by the spectral data. The results of antibacterial activity of these compounds were obtained against certain strains of Gram-positive and Gramnegative bacteria with reference to ciprofloxacin. The MIC values declared the most of the compounds good antibacterial agents and hence these can be further subjected to in vivo study along with cytotoxic study. Thus the series of molecules can be considered by the pharmacological industries as new drug candidates for the drug discovery pathway.

\section{ACKNOWLEDGEMENT}

The Higher Education Commission (HEC) of Pakistan is highly acknowledged by the authors for financial support regarding purchasing of chemicals and spectral study.

\section{REFERENCES}

1. P. C. Sharma, S. Jain, Acta Pharma. Sci. 50, 35 (2008)

2. P. C. Sharma, S. Jain, Acta Pol. Pharm. Drug Res. 65, 551 (2008)

3. Y. Q. Ge, J. Jia, T. Wang, H. W. Sun, G. Y. Duan, J. W. Wang, Spectrochim. Acta Mol. Biomol. Spectrosc. 123, 336 (2014)

4. K. Zhang, P. Wang, L. N. Xuan, X. Y. Fu, F. Jing, S. Li, Y. M. Liu, B. Q. Chen, Bioorg. Med. Chem. Lett. 24, 5154 (2014)

5. P. Li, L. Shi, M. N. Gao, X. Yang, W. Xue, L. H. Jin, D. Y. Hu, B. A. Song, Bioorg. Med. Chem. Lett. 25, 481 (2014)

6. M. M. G. El-Din, M. I. El-Gamal, M. S. Abdel-Maksoud, K. H. Yoo, C. H. Oh, Bioorg. Med. Chem. Lett. 25, 1692 (2015)

7. M. M. G. El-Din, M. I. El-Gamal, M. S. Abdel-Maksoud, K. H. Yoo, C. H. Oh, Eur. J. Med. Chem. 90, 45 (2014)

8. A. A. Othman, M. Kihel, S. Amara, Arabian J. Chem. http://dx.doi. org/10.1016/j.arabjc.2014.09.003 (2014)

9. J. Lian, Z. Qiang, M. Li, J. R. Bolton, J. Qu, Water Res. 75, 43 (2015)

10. T. A. Kung, C. W. Tsai, B. C. Ku, W. H. Wang, Food Chem. 175, 189 (2015)

11. M. Farahi, B. Karami, H. M. Tanuraghaj, Tetrahedron Lett. 56, 1833 (2015)

12. S. Konda, S. Raparthi, K. Bhaskar, R. K. Munaganti, V. Guguloth, L. Nagarapu, D. M. Akkewar, Bioorg. Med. Chem. Lett. 25, 1643 (2015)

13. C. C. Yang, C. L. Huang, T. C. Cheng, H. T. Lai, Biodegradation http:// dx.doi.org/10.1016/j.ibiod.2015.01.015 (2015)

14. M. M. Ghorab, F. A. Ragab, H. I. Heiba, M. G. El-Gazzar, S. S. Zahran, Eur. J. Med. Chem. 92, 682 (2015)

15. S. R. Khobare, V. S. Gajare, N. Rajana, R. Datrika, K. S. Reddy, U. K. S. Kumar, V. Siddaiah, Tetrahedron Lett. http://dx.doi.org/10.1016/j. tetlet.2015.02.042 (2015)

16. V. D. Vitnik, Z. J. Vitnik, Spectrochim. Acta Mol. Biomol. Spectrosc. 138, $1(2015)$

17. S. D. Castro, M. J. Camarasa, J. Balzarini, S. Velazquez, Eur. J. Med. Chem. 83, 174 (2014)

18. Aziz-ur-Rehman, A. Fatima, N. Abbas, M. A. Abbasi, K. M. Khan, M. Ashraf, I. Ahmad, S. A. Ejaz, Pak. J. Pharm. Sci. 26, 345 (2013)

19. Aziz-ur-Rehman, A. Fatima, M. A. Abbasi, S. Rasool, A. Malik, M. Ashraf, I. Ahmad, S. A. Ejaz, J. Saudi Chem. Soc. Doi:http://dx.doi. org/10.1016/j.jscs.2013.02.006 (2013)

20. M. Kaspady, V. K. Narayanaswamy, M. Raju, G. K. Rao, Lett. Drug Des. Discov. 6, 21 (2009)

21. C. R. Yang, Y. Zang, M. R. Jacob, S. I. Khan, Y. J. Zhang, X. C. Li, Antimicrob. Agents Ch. 50, 1710 (2006) 